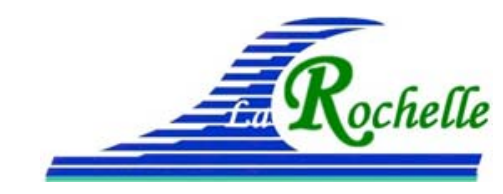

XVìmes Journées Nationales Génie Côtier - Génie Civil

La Rochelle, 29 au 31 mai 2018

DOI:10.5150/jngcgc.2018.062 C Editions Paralia CFL

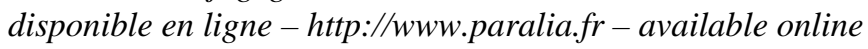

\title{
Étude multicritères de sites propices aux tests de prototypes hydroliens, de petites et moyennes puissances, dans la Mer des Pertuis
}

\section{Olivier LE MOINE ${ }^{1}$, Robin PASQUET ${ }^{2}$, Philippe GEAIRON ${ }^{1}$, Damien VOYER ${ }^{3}$, Audrey BRUNEAU ${ }^{1}$}

1. Laboratoire Environnement Ressource des Pertuis Charentais, Station Ifremer, 17390

La Tremblade, France.

Olivier.Le.Moine@ifremer.fr

2. CD17, Maison de la Charente Maritime, 85 boulevard de la République, 17076 La Rochelle, France.

robin.pasquet@charente-maritime.fr

3. École d'ingénieurs en génie des systèmes industriels, EIGSI, 26 rue de Vaux de Foletier, 17041 La Rochelle cedex 1, France.

\section{Résumé :}

Le développement des Energies Marines Renouvelables (EMR) fait partie des priorités mondiales ; il rentre également dans celles de l'Europe et de l'Etat Français. Le Conseil Départementale de la Charente Maritime (CD17), dans cette optique, et vu la situation privilégiée de son littoral, a décidé la mise en œuvre d'une étude sur la faisabilité d'installation de sites d'essais dédiés aux systèmes hydroliens. La bathymétrie et l'hydrodynamique analysées ont conduit à cibler les systèmes de petites à moyennes puissances. Dans un second temps, un panel de variables complémentaires (usages, règlementation, environnement, etc.) sont croisées afin de nuancer localement les sites réputés favorables par la sélection préalable à l'aide des variables d'altimétrie et de courant. Cette approche multicritère s'appuie sur la compilation de couches SIG pondérées suivant l'intérêt spécifique de la problématique. Les données sont de natures et sources diverses: données maillées continues ou sectorisations vectorielles, provenant de l'IGN, de l'Ifremer, de l'Etat, de l'ULR, du CD17, etc. Les principaux attendus sont: 1) une analyse spatiale complète de la Mer des Pertuis, précisant les zones ayant une dynamique intéressante pour les développeurs, 2) une caractérisation hiérarchisée des sites, base d'élaboration de la rédaction d'un catalogue qualitatif des sites et 3) l'anticipation d'éventuels conflits d'usages entre les acteurs de la zone et le projet. La première phase de développement des outils d'analyse spatiale et de sélection des sites est présentée ici.

\section{Mots-clés :}

Energies Marines Renouvelables, Hydrolien, Sélection de site, SIG, Modélisation hydrodynamique. 


\section{Thème 5 - Énergies et ressources marines}

\section{Introduction}

Le développement des énergies renouvelables est un objectif mondial, et prioritaire pour l'Union Européenne et l'Etat Français. L'utilisation de l'énergie des océans par les systèmes hydroliens est l'une des méthodologies alternatives possible et est étudiée mondialement. L'importance du littoral national et de la situation privilégiée de la Charente Maritime sur la façade Atlantique a conduit le Conseil Départemental de la Charente Maritime à analyser la faisabilité d'implantation de sites d'essais dans les Pertuis Charentais. Il s'appuie pour cela sur un environnement et un partenariat scientifique, d'ingénierie et industriel présent régionalement. Au vu des contraintes techniques des systèmes existants connus et de l'environnement géophysique et humain des Pertuis (bathymétrie, courants de marée, usages, etc), l'analyse a rapidement été orientée vers des systèmes de petites et moyennes puissances, ayant possiblement des exigences différentes.

L'objectif de cette étude est double et concerne la définition et la caractérisation des sites d'essais potentiels dans la Mer des Pertuis Charentais, en soutien au secteur industriel dans ses phases de développement et de tests. Cela doit permettre de 1) donner les éléments objectifs de choix de site en fonction du type d'appareil et 2) fournir pour chaque site potentiel le plus d'informations pratiques possibles pour aider à l'implantation. Les aspects technico-économiques (coût de raccordement, rendement,...) ne sont pris en compte que dans un second temps, au cas par cas, sur les sites sélectionnés dans d'autres études. S'agissant de sites d'essais, la nécessité de rendements élevés n'a pas forcément le même poids pour les éventuels utilisateurs industriels; la définition et la connaissance expérimentale de l'impact d'une machine sur le site n'étant pas à systématiquement corréler à son rendement sur le site choisi. Un Système d'Information Géographique (SIG) est utilisé pour analyser, classer et croiser les différentes informations. Outre sa capacité d'analyse spatiale, la faculté de représentation du SIG en fait un outil d'aide à la concertation locale, et d'anticipation d'éventuels conflits d'usages, ou d'évolution réglementaire nécessaire.

\section{Matériel et méthode}

La méthodologie d'analyse multicritère spatialisée utilisée est bien connue dans les études de sélection de sites pour l'aquaculture côtière (TELFER et al., 1998). En ce qui concerne l'hydrolien, elle a été déclinée sur le littoral Français par la Direction Générale de l'Energie et du climat (DGEC, 2013) du MEDDE. Elle se déroule en trois temps : 1) sélection des variables spatiales à prendre en compte, 2) reclassement de ces variables en fonction des contraintes techniques des différents types d'appareils, et 3) croisement de ces variables avec une pondération adaptée à la thématique. Le modèle d'estimation des courants est le modèle Mars3D de l'Ifremer, validé par ailleurs (LAZURE \& DUMAS, 2008). Le logiciels SIG utilisé est QGIS, complété du plug-in Octopus de traitement des données multidimensionnelles de sorties de modèle du type Network 


\section{XVèmes Journées Nationales Génie Côtier - Génie Civil \\ La Rochelle, 29 au 31 mai 2018}

Common Data Form (NetCDF (C) ULR - Ifremer). Les autres traitements sont faits sous MatLab, Les variables sélectionnées sont de deux types, soit exclusives (de type binaire, caractérisant la possibilité oui/non), soit de caractérisation, quantifiant la qualité du site (plus ou moins favorables).

Seules les variables exclusives sont utilisées dans la phase de sélection. Elles sont de format et origines très diverses; les variables continues, comme la bathymétrie ou l'intensité des courants, par exemple, sont utilisées après choix motivé des seuils d'exclusion à appliquer (hauteur d'eau résiduelle aux plus basses mers et diamètre estimé des machines, ou vitesse de courant nécessaire à la mise en route de l'appareil, par exemple). Les variables de secteurs définis, de type vectoriel, permettent d'exclure les zones où les usages sont complètement incompatibles avec l'implantation de systèmes. Il s'agit des zones d'attentes ou de circulation des cargos vers les ports de La Rochelle et Rochefort, des emprises d'élevages conchylicoles, des zones portuaires, ou de servitudes particulières, existence de câbles sous-marins ou d'épaves par exemple. La nature géologique des fonds peut également déterminer des zones d'exclusion; des fonds sableux, très mobiles sous des intensités de courant élevées peuvent s'avérer problématiques (cas du Pertuis de Maumusson par exemple). La diversité de la nature et format de toutes ces variables se retrouve dans celle de leurs sources : IGN, SHOM, DDTM, CD17, IFREMER, etc.

Les variables continues nécessaires à la sélection, comme la qualification des courants sur la colonne d'eau (variables à 4 dimensions), nécessitent des traitements particuliers avant représentation cartographique. Les vitesses de courant sont représentées soit par des estimations de leur permanence au cours du temps (pourcentage du temps où ils sont supérieurs au seuil préalablement défini), soit par l'estimation des puissances disponibles.

Toutes les autres variables, dites de caractérisation du site et non-exclusives, sont ensuite étudiées dans la description précise du site au cas par cas. Elles doivent apporter l'ensemble de l'information pour orienter les candidats sur les choix de techniques d'implantation, d'élaboration des coûts d'installation, d'études ou précautions préalables nécessaires vis-à-vis des zonages environnementaux. Il s'agit en l'occurrence de l'éloignement des postes de raccordement, de la nature des sols (dureté, portance, etc) pour l'infrastructure, des différents zonages ( Natura 2000, ZNIEFF, sites inscrits, etc) pour prescriptions particulières.

L'étude se focalise sur des systèmes hydroliens de "petites" et "moyennes" puissances, gammes peu développées aujourd'hui. De nombreux auteurs traitent de la caractérisation de sites hydroliens, mais dans le cadre de systèmes produisant des puissances nominales de plusieurs centaines de kilowatts et d'une utilisation commerciale (GOOSH et al., 2009 ; RAHIMI et al.,2014). Un des enjeux de cette étude réside dans la nécessité de caractériser un site sans contraindre les critères de recherche 


\section{Thème 5 - Énergies et ressources marines}

à une technologie précise, tout en utilisant des seuils cohérents en termes de "petites" et "moyennes" puissances.

Une méthode précise de caractérisation de site est décrite dans le document technique de l'EMEC, European Marine Energy Center Ltd (LEGRAND, 2009). Il y est introduit la notion de densité de puissance moyenne électrique APD ("Average Power Density") $\left[\mathrm{W} / \mathrm{m}^{2}\right]$, une grandeur caractérisant la puissance unitaire extractible par une hydrolienne, ayant pour variables l'intensité du courant $U_{i}$ et les occurrences de courant $f\left(U_{i}\right)$ selon les formules (1) et (2). La masse volumique de l'eau salée $\rho_{e}$ est fixée à $1025 \mathrm{~kg} / \mathrm{m}^{3}$. On fixe $C p$, le rendement de la machine à 0,35 , conformément aux informations échangés avec des développeurs de systèmes de petit hydrolien (i.e : Ecocinetic). En cas, de caractérisation d'un site pour une machine définie, il sera plus judicieux de prendre le $C p$ fourni par le développeur.

$A P D_{\text {hydro }}=\frac{1}{2} \cdot \rho_{e} \sum_{i=1}^{N_{B}}\left(U_{i}^{3} \cdot f\left(U_{i}\right)\right)$

$A P D_{\text {élec }}=C_{p} \cdot A P D_{\text {hydro }}$

où $\mathrm{NB}$ est le nombre d'intervalles $[\mathrm{Ui}-\Delta \mathrm{U} / 2 ; \mathrm{Ui}+\Delta \mathrm{U} / 2]$ inclus dans $[0 ; \mathrm{Umax}]$.

Dans le document de l'EMEC, $\Delta U=0,1 \mathrm{~m} / \mathrm{s}$. Cependant, l'auteur du document de l'EMEC ne se positionne pas précisément sur les seuils de densité acceptables, notamment pour la mise en place d'un bassin d'essai hydrolien. De plus, les puissances de machines évoquées sont de l'ordre de plusieurs centaines de kilowatts, à but de production industrielle $(500 \mathrm{~kW}$ de puissance pour une turbine de $22 \mathrm{~m})$. Faute de références spécifiques et de définition de gammes de puissance précises, il a fallu déterminer des intervalles cohérents avec cette nomenclature de terminologie "petite" et "moyenne" puissance, et y rattacher les densités de puissances correspondantes. Pour cela, un parallèle a été fait avec l'éolien, technologie ayant développée des systèmes de puissances équivalentes, certaines entreprises décrivant des produits allant de la centaine de watts à une centaine de kilowatt (mini-éolien à moyen éolien). Nous proposons d'adopter cette nomenclature et d'en déduire les valeurs de densités de puissances des futurs systèmes hydroliens. Une fois ces intervalles de puissance définis, il nous a été possible de fixer les seuils altimétriques à prendre en compte, en s'appuyant sur la documentation technique d'hydroliennes existantes, puis d'en déduire un intervalle de densité de puissance électrique correspondant à chaque gamme de puissance.

En conséquence, pour décrire plus précisément les sites acceptables d'un point de vue hydrodynamique, nous avons donc choisi de 1) proposer une définition de la nomenclature générique de "petites" et "moyennes" puissances en s'appuyant sur les gammes existantes, 2) de faire le parallèle entre puissances nominales éolien et hydrolien et renseigner l'emprise associée à chacune, 3) décrire les densités de puissance nominales électriques ainsi que les contraintes bathymétriques correspondant à chaque gamme, 4) estimer les seuils de densité de puissance hydrodynamique et 


\section{XVèmes Journées Nationales Génie Côtier - Génie Civil \\ La Rochelle, 29 au 31 mai 2018}

bathymétrique limites 5) rechercher les sites validant les seuils fixés. Une seconde phase de caractérisation précise est effectuée sur les sites validant les hypothèses précédentes.

\section{Résultats}

Les gammes de puissance ont été définies suivant les gammes d'utilisations potentielles, exprimées en nombre de foyers. La puissance électrique moyenne consommée par un foyer a été estimée à $6 \mathrm{~kW}$, en considérant que la majorité des foyers français sont raccordés au réseau via des disjoncteurs de 6kVA. Trois intervalles de puissance sont proposés, faisant écho aux gammes éoliennes (ENERGIE PLUS). L'emprise des machines $D$ a été estimée à la suite d'une recherche sur les documents techniques d'hydroliennes existantes. Les densités de puissance ont été calculées grâce à la formule (3) en considérant la surface balayée $A$ comme un disque de diamètre $D$, surface de récupération d'énergie.

$P D_{N}=P_{N} / A$

où $\mathrm{PDN}_{\mathrm{N}}$ : Densité de Puissance Nominale, $\mathrm{P}_{\mathrm{N}}$ : Puissance nominale, $\mathrm{A}=\pi \cdot(\mathrm{D} / 2)^{2}$ : aire de récupération d'énergie correspondant à la gamme de puissance.

Ces valeurs sont théoriques, issues de travaux bibliographiques et nécessitent des études expérimentales afin d'être validées.

Tableau 1. Proposition de nomenclature en fonction des puissances de machine.

\begin{tabular}{|l|l|l|l|l|}
\hline Terminologie & $\begin{array}{l}\text { Nombre de foyers } \\
\text { équivalent }(\mathbf{6 k W})\end{array}$ & $\begin{array}{l}\text { Puissance } \\
\text { nominale }\left(\boldsymbol{P}_{\mathrm{N}}\right)\end{array}$ & $\begin{array}{l}\text { Emprise des } \\
\text { hydrolienne } \\
\text { s (D) }\end{array}$ & $\begin{array}{l}\text { Densité de puissance } \\
\text { nominale équivalente } \\
\left(\mathbf{P D}_{N}\right)\end{array}$ \\
\hline Micro hydrolien & Foyer isolé & $1 \mathrm{~kW}-5 \mathrm{~kW}$ & $1 \mathrm{~m}$ à $3 \mathrm{~m}$ & $140 \mathrm{~W} / \mathrm{m}^{2}$ à $1,59 \mathrm{~kW} / \mathrm{m}^{2}$ \\
\hline Petit hydrolien & $\begin{array}{l}\text { Groupes de } 2 \text { à } 3 \\
\text { foyers }\end{array}$ & $5 \mathrm{~kW}-20 \mathrm{~kW}$ & $2 \mathrm{~m}$ à $5 \mathrm{~m}$ & $255 \mathrm{~W} / \mathrm{m}^{2}$ à $6,10 \mathrm{~kW} / \mathrm{m}^{2}$ \\
\hline Moyen hydrolien & $\begin{array}{l}\text { Petite entreprise } \\
\text { ou 4 à 15 foyers }\end{array}$ & $20 \mathrm{~kW}-100 \mathrm{~kW}$ & 3 à $6 \mathrm{~m}$ & $700 \mathrm{~W} / \mathrm{m}^{2}$ à $14,1 \mathrm{~kW} / \mathrm{m}^{2}$ \\
\hline
\end{tabular}

Pour lier les intervalles de densités de puissances nominales $P D D_{\mathrm{N}}$ aux densités de

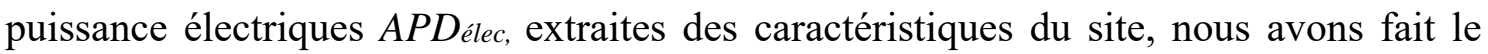
choix d'introduire la grandeur $\eta_{\text {site, }}$ l'optimalité du site. Elle correspond au rapport de la densité de puissance moyenne électrique mesurée sur une machine mise sur site par la puissance nominale de cette même machine $\left(A P D^{\exp }{ }_{e ́ c} / P_{N}\right)$. Elle a été évaluée selon SARKAR \& BEHERA (2012), traitant des systèmes éoliens. Pour un site éolien qualifié de bon, la puissance fournie moyennée sur un an s'élève à environ $35 \%$ de la puissance nominale de l'éolienne,

soit $\eta_{\text {site }}=0,35$. Nous avons fait le choix de transposer cette valeur à la technologie hydrolienne.

Les cartes de densités de puissances électriques ont été calculées à l'aide du logiciel MatLab et des simulations issues de Mars 3D via la formule (2). Le seuil limite de 


\section{Thème 5 - Énergies et ressources marines}

densité de puissance $A P D \lim$ a été calculé grâce à la formule (4) pour obtenir la valeur de $50 \mathrm{~W} / \mathrm{m}^{2}$.

$A P D_{\text {lim }}=\eta_{\text {site }} \cdot \operatorname{Min}\left(\left|P D_{\mathrm{N}}\right| P_{N}\right)$

Nous avons fait le choix de fixer le seuil bathymétrique à environ deux fois l'emprise maximale du système. Cela assure l'immersion des systèmes pour des états de mer forte observés dans les Pertuis. L'analyse spatiale a été réalisée sur les données de courant à mi-hauteur de la colonne d'eau. Deux zones sont repérées sur la figure 1, chacune correspondant à un seuil. La première traite de la permanence du courant, soit les zones où la vitesse des courants est supérieure ou égale à $0,5 \mathrm{~m} / \mathrm{s}$ pendant $50 \%$ du temps. Le seuil de vitesse a été choisi égal à la vitesse de démarrage théorique d'une hydrolienne (LEGRAND, 2009) et la valeur de 50\% du temps a été estimée suffisante pour étudier l'impact de l'hydrolienne sur son milieu (sans garantie de production électrique significative cependant). La seconde correspond aux secteurs où la densité de puissance moyenne électrique $\left(A P D_{e ́ l e c}\right)$ est supérieure ou égale à $50 \mathrm{~W} / \mathrm{m}^{2}\left(A P D_{\text {lim }}\right)$, estimée fournir une production significative (figure 1).

La zone potentielle de l'embouchure de la Charente, comme celle située dans le port de la Pallice à La Rochelle, se trouve exclue par la circulation de cargos à fort tirant d'eau, celle de la Sèvre Niortaise par les hauteurs d'eau insuffisantes au regard du transit de bateaux professionnels (figure 1). La zone d'embouchure de la Seudre, au fonctionnement hydraulique sous influence d'une superficie importante de marais salés, fait l'objet d'une étude spécifique. Les zones vérifiant le seuil $A D P$ élec $>A P D_{\text {lim }}$ sur l'ensemble de la Mer des Pertuis ne représentent alors que 6,2\% de sa superficie et concernent principalement quatre sites : l'un situé au nord de l'île d'Oléron, le second dans le pertuis de Maumusson, le troisième au sud de la pointe du Grouin du Cou et le dernier à proximité du pont reliant l'île d'Oléron au continent. Sur chacun d'eux, une caractérisation plus détaillée permettra de cerner plus précisément les éventuelles limites propres à chaque zone.

\section{Conclusions}

L'étude multicritères est assez délicate à mener, s'agissant d'un secteur en cours de développement, et aux spécifications techniques encore mal définies. Une méthodologie a néanmoins été développée, pour évaluer les divers seuils à prendre en compte et représenter cartographiquement les capacités théoriques de l'environnement à soutenir des systèmes hydroliens.

L'étendue des zones à potentiel hydrolien significatif ne représenterait selon notre étude que $6,2 \%$ de la superficie analysée de la Mer des Pertuis. L'analyse montre que les surfaces où les courants dépassent $0,5 \mathrm{~m} / \mathrm{s}$ sur $50 \%$ du temps sont un peu plus importantes $(8,3 \%$ de la superficie totale), mais n'engendreraient pas de production très significative dans l'état actuel des connaissances. 


\section{XVèmes Journées Nationales Génie Côtier - Génie Civil \\ La Rochelle, 29 au 31 mai 2018}

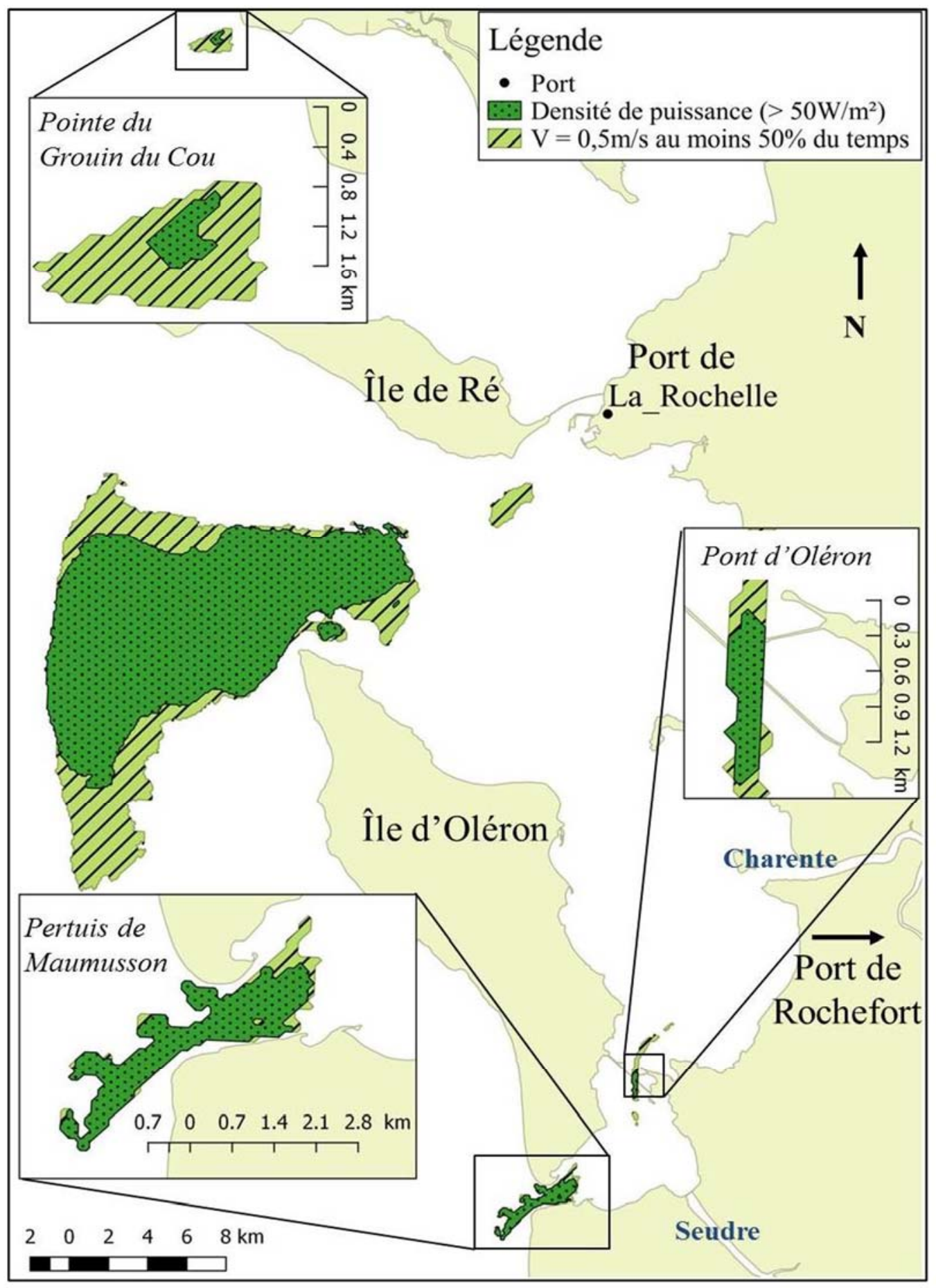

Figure 1. Sites satisfaisant aux critères de sélection de densité de puissance.

Ces résultats sont à valider expérimentalement, notamment les seuils de densités de puissance nominales et les rendements de sites afin de valider et affiner les traitements géostatistiques. 


\section{Thème 5 - Énergies et ressources marines}

Les effets de la houle n'ont pas été pris en compte ici, bien qu'ils puissent jouer un rôle non négligeable selon les secteurs considérés.

Ces résultats ne concernent que la sélection de sites et seront, dans la suite du projet, à pondérer en fonction des contraintes particulières de chaque site sélectionné. Cela sera fait lors de l'établissement des diverses fiches de caractérisation des sites.

Remerciements : Les auteurs tiennent à remercier le Conseil Départemental de Charente Maritime qui finance ces travaux.

\section{Références bibliographiques}

DGEC-DIRECTION GENERALE DE L'ENERGIE ET DU CLIMAT. (2013). Etude multicritère pour la définition du potentiel hydrolien en France métropolitaine. Ministère de l'Ecologie et de Développement Durable. URL: https://www.cerema.fr/fr/system/files/production/2017-12/resume_hydrolien.pdf

ENERGIE PLUS (Site web). https://www.energieplus-lesite.be

GOOSH S., THOMSON J., POLAGYE B., MEGGIT D. (2009). Site characterization for tidal power. MTS/IEEE Biloxi - Marine Technology for Our Future: Global and Local Challenges, OCEANS.

SARKAR A., BEHERA D.K. (2012). Wind turbine blade efficiency and power calculation with electrical analogy. International Journal of Scientific and Research Publications, Vol. 2(2).

LAZURE P., DUMAS F. (2008). An external-internal mode coupling for a 3D hydrodynamical model for applications at regional scale (MARS). Advances In Water Resources, Vol. 31(2), pp 233-250. https://doi.org/10.1016/j.advwatres.2007.06.010

LEGRAND C. (2009). Assessment of tidal energy resource. The European Marine Energy Centre Ltd. URL : http://www.emec.org.uk/

RAHIMI R., PENESIS I., HEMER M., MASON L., THOMAS G. (2014). Characterisation of the tidal current resource in Tasmamnia, AWTEC 2014, 2nd Asian wave and tidal energy conference.

TELFER T., ROSS L. G., BEVERIDGE M. (1998). Use of GIS for coastal aquaculture site selection and environemental management. Aquaculture '98 Book of Abstracts, 536. World Aquaculture Society, 143 J. M. Parker Coliseum Louisiana State University, Baton Rouge, LA 70803 USA, 1998. 